LSM $\geq 10-15 \mathrm{kPa}$ and $124 / 557$ patients (22.3\%) with a LSM $>15 \mathrm{kPa}$ were identified as having regular surveillance for HCC.

Conclusions This study shows that information routinely collected in EHR can be used to evaluate screening and surveillance strategies in patients with cirrhosis. Only a minority of patients had undergone the recommended interventions within the definitions of this study. This highlights the potential for a cirrhosis registry to impact on patient care and to improve outcomes for patients with cirrhosis.

\section{PWE-090 WEST MIDLANDS MULTI-CENTRE TRAINEE-LED AUDIT IN THE ASSESSMENT, MANAGEMENT AND PROPHYLAXIS OF SPONTANEOUS BACTERIAL PERITONITIS}

Keith Siau*, B Hicken, A McCulloch, M Harborne, T Gupta, B Polewiczowska, T Troth, M McFarlane, E Mozdiak, A Thakor, Z Rehman, L O'Flynn, Monika Widlak. (West Midlands Research in Gastroenterology), UK

\subsection{6/gutjnl-2018-BSGAbstracts.232}

Introduction Spontaneous bacterial peritonitis (SBP) is a common but potentially fatal complication in patients with cirrhosis and ascites. In the first audit performed by West Midlands Research in Gastroenterology (WMRIG) trainees, we aimed to assess practice of assessment, management and primary and secondary prophylaxis of SBP according to national standards, in addition to the feasibility of regional project delivery.

Methods This trainee-led, retrospective, multi-centre study identified patients admitted with cirrhosis and ascites between Sep-Dec 2016. Outcomes of SBP and mortality were retrospectively followed-up for up to 1 year (median 8 months). Practice was audited against EASL, BSG and NICE standards. Heterogeneity between sites was assessed with chi $^{2}$ and timeto-event analyses undertaken using Kaplan-Meier plots.

Results Trainees across 8 West Midlands hospitals identified 227 patients (mean age 58, SD 13; 65\% male) with 282 admissions. Cirrhosis was attributed to alcohol (79\%), NAFLD (10\%), autoimmune $(4 \%)$ and viral $(3 \%)$, and was graded Child-Pugh B in $48 \%$ and $\mathrm{C}$ in $49 \%$. 18\% were elective admissions and $7 \%$ had a previous history of SBP. Ascitic aspirates were performed in 83\% (range: 60\%-92\%, $\mathrm{p}=0.019$ ), in $<24$ hours in 64\% (range: $49 \%-85 \%, \mathrm{p}=\mathrm{NS}$ ), and cultures sent in 55\% (range: $11 \%-86 \%, \mathrm{p}<0.001$ ). 16.8\% of aspirates met criteria for SBP: antibiotics were commenced in $92 \%$ $(\mathrm{p}<0.001)$, Day 1 albumin in 64\% ( $=\mathrm{NS})$, Day 3 albumin in $40 \%(\mathrm{p}=\mathrm{NS})$, and secondary prophylaxis in $44 \% \quad(\mathrm{p}=\mathrm{NS})$. Repeat aspirate to ensure SBP resolution was performed in 33\% (range: $0 \%-52 \%, \mathrm{p}=\mathrm{NS}$ ). In patients without SBP, ascitic protein was measured in $46 \%$ (not available in 3 Trusts). 32 $(67 \%)$ met criteria for primary prophylaxis (protein $\leq 15 \mathrm{~g} / \mathrm{L}$ ); which was commenced in $4(13 \%$, range $0 \%-100 \%, p=N S)$. Mortality occurred in 51\%. SBP was associated with lower median survival (79 days vs. non-SBP: 190 days, $p=0.045$ ) [figure 1]. Emergency admission (HR 8.4, $p=0.039$ ), older age (HR 1.03 per increase, $\mathrm{p}=0.017$ ) and ascitic protein $\leq 15 \mathrm{~g} / \mathrm{L}$ (HR 2.27, $\mathrm{p}=0.042$ ) were multivariate predictors of reduced survival. SBP occurred after discharge in 8 patients (4\%) after a median interval of 32 days.

Conclusions Our pilot study has been successful in highlighting deficiencies and variations in the assessment, management and prophylaxis of SBP. These Results will inform and prioritise future regional quality improvement strategies to improve outcomes in patients with advanced chronic liver disease.

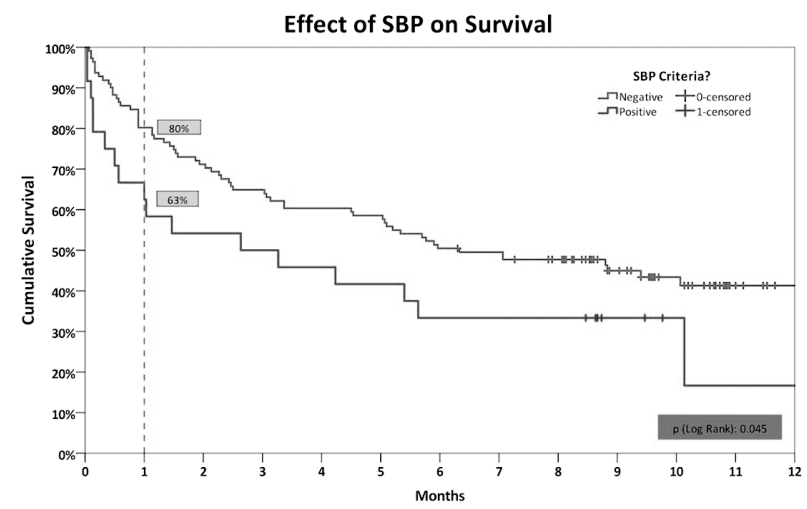

Abstract PWE-090 Figure 1 Effect of SBP on survival

\section{PWE-091 L-ORNITHINE L-ASPARTATE IN MINIMAL HEPATIC ENCEPHALOPATHY: POSSIBLE EFFECTS ON THE BRAIN- MUSCLE AXIS?}

Y Pasha*, S Taylor-Robinson, R Leech, I Ribeiro, N Cook, M Crossey, H Marcinkowski. Imperial College London, London, UK

\subsection{6/gutjnl-2018-BSGAbstracts.233}

Introduction L-Ornithine L-Aspartate (LOLA) is an ammonialowering agent for treatment of hepatic encephalopathy (HE); it may reduce sarcopenia. We investigated 12 weeks of oral LOLA in patients with compensated cirrhosis and minimal HE (MHE).

Methods Consecutive patients were pre-screened with paperand-pencil-based psychometric testing (PHES test) and included if performance was impaired to a level of -4 or worse. 34 English-speakers were included; 12 randomised to 12 weeks oral LOLA $6 \mathrm{~g}$ tds, 22 randomised to identical-looking placebo. At baseline, 4 and 12 weeks, subjects had PHES, a computerised battery: Cogstate ${ }^{\mathrm{TM}}$ and SF-36 health questionnaires. Markers of muscle function were recorded: handgrip strength, skin fold thickness, and 6-minute-walk-test. Subjects had cerebral $\mathrm{T}_{1}$ and $\mathrm{T}_{2}$ MRI, functional MRI (fMRI) with motor/cognitive tasks/resting state studies; and ${ }^{1} \mathrm{H}$ MRS. LC Model software was used for metabolite identification.

Results On SF-36, 57\% on LOLA reported better energy levels than placebo $0.04 \%$ (P-value $<0.001$ ). Better concentration was reported by $21 \%$ in treatment arm vs none in placebo group $(\mathrm{p}=0.05) .28 \%$ reported improved memory in treatment group vs $0.04 \%$ with placebo. Sleep improvements were reported by $35 \%$ in treatment arm vs $0.09 \%$ on placebo $(\mathrm{p}=0.05)$. In both groups, changes in total PHES score and Cogstate $^{\text {TM }}$ were non-significant. PHES test sub-analysis of the Digit-Symbol showed significant improvement in performance in LOLA-treated group $(p=0.05)$. Biceps skinfold thickness showed a mean gain of $1.5 \mathrm{~mm}$ in the LOLA group with mean loss of $1.0 \mathrm{~mm}(\mathrm{p}=0.05)$ in placebo. No differences were found in other skinfolds, hand-grip or 6-minute-walktest. On $\mathrm{T}_{1}$ cerebral MRI, significant volume reduction was seen in left lateral ventricle, right globus pallidus and midanterior corpus callosum (ACC). fMRI tasks did not vary between groups. ${ }^{1} \mathrm{H}$ MRS of ACC showed significant changes in glutamate concentration $(p=0.03)$, after LOLA. 
Conclusion After 12 weeks LOLA, patients reported highly significant improvement in energy levels and concentration. Although 12 weeks LOLA had no overall effect on psychometric performance, significant treatment-related improvement in digit-symbol PHES subtest in those receiving LOLA was seen. An increase was noted in biceps skinfold thickness, which may indicate improved nutrition. Subcortical brain areas showed volume reduction, an observation not previously noted in imaging studies of patients receiving this drug. Unlike previous studies, no functional changes were seen, but significant changes were found on MRS of ACC, a region known to be metabolically active in mHE. It may be that a larger dose of LOLA would have shown greater effects on psychometric performance.

\section{PWE-092 A STRATIFIED HOME BASED CARE PATHWAY FOR THE TREATMENT OF HCV INFECTION}

Brian Thomson*, Jasmina Khaldi, Samantha Bird, Tom Bills, Breanne Dilks, Stephen Ryder. Nottingham University Hospitals, Nottingham, UK

\subsection{6/gutjnl-2018-BSGAbstracts.234}

Introduction The advent of directly acting anti-viral agents (DAAs) for HCV infection has transformed the therapeutic landscape. DAAs have an excellent safety record, and can be stratified for use in community environments which better meet patient needs. We present a pilot study of a novel home based care pathway, delivered by a partnership between the local Operational Delivery Network (ODN), specialist hepatitis services and clinical Pharmacy.

Methods HCV infected patients assessed at the ODN multidisciplinary team meeting (MDT) as eligible for DAAs were screened for entry to the study by the following criteria: competence to adhere to therapy without direct supervision; no current or previous evidence of hepatic decompensation; contactable by telephone. Following recruitment, patients received a pack containing: blood forms; a schedule for community blood testing; information leaflets; details of hospital contacts. DAAs were delivered to the patient home each month by Pharmacy. A dedicated Pharmacy technician acted as initial contact for patient queries, with support from the lead nurse specialist. The homecare technician worked closely with the nursing team, the MDT co-ordinator and ODN Manager to co-ordinate the service. Lab Results were reviewed by the lead nurse. Patients were invited to report outcome measures and feedback using structured questionnaires.

Results Of 121 patients offered a choice of home or hospital based care by January 2018, 97 (80\%) elected to receive treatment at home. This group did not differ significantly in age, gender, HCV genotype or choice of DAA from those treated in the hospital clinic. 14 homecare patients met Fibroscan criteria for cirrhosis. Of the 97 patients so far started on treatment, 57 have completed, 31 achieved SVR and 4 have failed treatment. Three patients withdrew from the study for reasons unrelated to homecare and one transferred back to hospital care. 18 feedback questionnaires have been received from 56 so far sent (32\%). All respondents stated that the service had lived up to or exceeded expectations, and was particularly valued by patients living distant from the hospital. Conclusions Homecare provides a safe, transferable and scaleable treatment option which is preferred by patients. The strategy of pharmacy based implementation and economies of staff time intrinsic to the homecare model will relieve pressure on hepatitis services, and allow specialist teams to focus on patients with severe co-morbidities and promoting models for case finding and community care for harder to reach groups with HCV infection.

\section{PWE-093 TREATMENT OF GASTRIC FUNDAL VARICES WITH EUS GUIDED EMBOLISATION COMBINING COIL PLACEMENT WITH THROMBIN INJECTION}

${ }^{1} J o a n n e$ Orourke* ${ }^{1,2} \mathrm{Chander}$ Shekhar, ${ }^{1}$ Dhiraj Tripathi, ${ }^{1} \mathrm{Colm}$ Forde, ${ }^{1}$ Brinder Mahon. ${ }^{1}$ Queen Elizabeth Hospital, Birmingham, UK; ${ }^{2}$ Manor Hospital, Walsall, UK

\subsection{6/gutjnl-2018-BSGAbstracts.235}

Introduction Gastric varices are present in 5\%-33\% of patients with portal hypertension with incidence of bleeding of around $25 \%$ in 2 years. ${ }^{1}$ If gastric varices are identified as the source of bleeding, therapeutic options include endoscopic Methods, TIPSS, surgery and non-selective beta blockade. ${ }^{2}$ There are reports of EUS guided coiling combined with cyanoacrylate glue ${ }^{3}$ but limited literature on safety and efficacy of EUS guided coil embolisation with human thrombin injection. We report our experience.

Methods We analysed data of all EUS guided interventions for the management of bleeding gastric varices between 20152017 at a liver transplant centre. Olympus EUS linear scope was used to inject human thrombin (Tisseel; 500IU/ML) in gastric varices with or without coils (Nester Embolization Coils).

Results A total of 10 EUS guided interventions in 6 patients (4 $\mathrm{M}$ and $2 \mathrm{~F}$ ), aged 55 (41-59) yrs for secondary prophylaxis. $67 \%$ patients had cirrhosis with MELD score of 14(1021) and $75 \%$ were Child-Pugh class C. The remainder had non-cirrhotic portal hypertension. All patients had previous bleeding from gastric varices and $2 / 3$ rd were intolerant of beta-blockers. $67 \%$ had previous thrombin injection that had failed to obliterate the gastric varices. EUS guided coil embolisation was undertaken with thrombin injection in 6 , and thrombin alone in 4 ( 2 had previous coils embolisation). The largest feeding vessel was $12(7-16) \mathrm{mm}$ with a median 5 (210) coils placement followed by thrombin injection of 3500 (2500-5000) IU.

Most (8/10) stayed overnight after intervention and only 2 required longer stays, Median F/U was 9 (3-20) months with zero 30 day mortality. 1 patient had fever 2 days post procedure requiring IV antibiotics. No reported episodes of rebleeding except in 1 patient at 23 months. 4 had follow up EUS (5-7 months) and showed no flow at the level of the coils. 1 patient died within 3 months of procedure secondary to hepatic decompensation.

Conclusions In our experience EUS guided coil embolisation and injection of thrombin, is a technically safe and well-tolerated procedure even in patients with advanced liver disease especially who have failed eradication of gastric varices from single modality therapy. Due to the lower incidence of gastric variceal bleeding in comparison to oesophageal varices bleeding, we recommend multi-centre prospective data collection evaluating the modalities being used and reporting of outcomes to help inform national guidelines.

\section{REFERENCES}

1. Hepatology 1992;16:1343-1349.

2. Gut 2015;64:1680-704.

3. Gastrointest Endosc. 2016 Jun;83(6):1164-72. 